\title{
Apport de l'exploration radiographique tridimensionnelle de la région antérolatérale mandibulaire en chirurgie orale et implantaire.
}

\author{
Belhassaini $\mathbf{M}^{1}$, Colard T11, Nawrocki L1', Savignat $\mathbf{M}^{1}$ \\ 1. Service d'Odontologie - CHRU Lille
}

Introduction

La région antérolatérale mandibulaire est une région fréquemment explorée en chirurgie orale et implantaire. Bien que le risque chirurgical soit couramment considéré comme faible dans ce secteur, des complications d'ordre neurologique et hémorragique ont été rapportées. En effet, cette région anatomique est traversée par des éléments vasculaires (les artères sublinguale et submentale) et nerveux (les nerfs mentonnier et incisif), pouvant être difficilement identifiables à partir des clichés radiographiques conventionnels. L'objectif de ce travail est de faire une mise au point de la littérature sur l'apport de la radiographie tridimensionnelle (3D) par tomodensitométrie (TDM) et tomographie volumique à faisceau conique (CBCT) de la région antérolatérale mandibulaire, en particulier en chirurgie orale et implantaire.

\section{Matériels et méthodes}

À partir d'une recherche bibliographique de la base de données MEDLINE sans limite de période, les articles traitant de l'exploration radiographique de la région antérolatérale mandibulaire par TDM et CBCT ont été étudiés. L'analyse s'est particulièrement intéressée aux éléments anatomiques majeurs : le foramen mentonnier (FM), la boucle antérieure (BA) du pédicule mentonnier, le canal incisif mandibulaire $(\mathrm{Cl})$, les foramens et canaux linguaux mandibulaires (FL et $\mathrm{CL}$ ). 43 articles pertinents ont été étudiés.

\section{Résultats}

Les éléments anatomiques de la région antérolatérale mandibulaire ont été objectivés avec précision grâce à limagerie tridimensionnelle. Concernant le FM, les études ont révélé sa présence dans $95 \%$ des cas. Il a été situé coronairement aux apex des prémolaires dans 25 à $38 \%$ des cas. Des FM accessoires ont été retrouvés dans $3 \%$ des cas. La BA a été identifiée dans $90 \%$ des cas, avec une longueur moyenne égale à 2,6 mm. Les FL et CL ont été dépistés sur $94 \%$ des images radiographiques tridimensionnelles, la présentation la plus classique ayant été la présence de $2 \mathrm{CL}$ sur la ligne médiane. Enfin, concernantle $\mathrm{Cl}$, sa présence a été déterminée dans $93 \%$ des cas, avec une longueur moyenne de $9 \mathrm{~mm}$.

\section{Discussion}

Les résultats de ce travail montrent l'apport incontestable de l'imagerie 3D par rapport à l'imagerie 2D en terme de sensibilité et de spécificité diagnostique. Ceci a été confirmé entre autre par la mise en évidence de variations anatomiques rares.

\section{Conclusion}

La boucle antérieure du pédicule mentonnier, le foramen mentonnier, le canal incisif mandibulaire, les foramens et canaux linguaux mandibulaires sont autant d'obstacles anatomiques livrant passage aux pédicules neurovasculaires dont le praticien doit tenir compte devant tout acte de chirurgie orale et implantaire. Le risque de complications hémorragique et neurologique dues aux lésions de ces éléments pourrait être minoré par une analyse préopératoire rigoureuse des clichés tridimensionnels, notamment à l'aide d'un guide radiologique.

belhassaini.mina@gmail.com

Bibliographie

Juodzbalys G et al. J Oral Maxillofac Res. 2010;1(1):e3

Imada TSN et al. Clin Oral Implants Res. 2014;25(2):e94-9.

Gómez-Román G et al. Implant Dent. 2015;24(5):578-85.

(C) The authors, published by EDP Sciences. This is an Open Access article distributed under the terms of the Creative Commons Attribution License 4.0 (http://creativecommons.org/licenses/by/4.0/). 